\title{
A New Speed Illusion: Blind Illusion
}

\author{
Yining Tong, Hiroyoshi Todo, Jian Sun, Xiaoxiao Qian
}

\begin{abstract}
Visual illusion is a specific perception that is inconsistent with the objective fact formed by humans or animals selecting improper reference or basing on perceptual experience when they observe objects. It reflects perceived ability and mechanism of visual system from a unique perspective. Therefore, it is of great significance to study illusion theory, establish proper mathematical models, and simulate natural scenes in computer for the development of psychology, physiology and computer vision. Illusions that can be quantitatively measured and quantitatively analyzed, is desired. In this paper, we utilize a new quantitative technique developed by our laboratory to measure a new blind illusion. The results can be expected to the elucidation of the mechanism of visual illusions, as well as leading to the understanding of perceptual features and intelligence.
\end{abstract}

Index Terms-Visual illusion, blind illusion, quantitatively measure, the mechanism of visual illusions.

\section{INTRODUCTION}

Elucidation of human intelligence is one of the greatest challenges to mankind in the 21st century. And the perceived ability to recognize the environment is one of the typical aspects of intelligence. It is the phenomenon which disturbs this perceived ability, features on the perception in an extreme form, appeared as the dare leads to the understanding of the fundamental features of perception, has recently been considered as an important method to study the neural mechanisms of visual perception and so as to human intelligence [1]-[3].

Many illusions have been observed and studied for a long time. One of the most famous illusions is 'wagon wheel illusion' [4] that is the apparent backwards rotation of a wheel that is in fact rotating forwards.

Fraser and Wilcox presented a motion illusion called an 'escalator illusion' that the luminance gradient reverses within a small region in many places produced an illusory motion [5]. Faubert, J. and Herbert presented an illusion related to the 'escalator illusion'. They proposed that the reversals in the luminance gradient result in changes in the direction of illusory motion, such that both clockwise and counterclockwise rotation can be observed in the same figure [6].

The complexity of visual perception has made people's elucidation different from each other, which is visual illusion.

\footnotetext{
Yingning Tong, Faculty of Engineering, University of Toyama, Toyama-shi, 930-8555 Japan

Hiroyoshi Todo, Wicresoft Japan Inc., Japan

Jian Sun, Faculty of Engineering, University of Toyama, Toyama-shi, 930-8555 Japan

Xiaoxiao Qian, Faculty of Engineering, University of Toyama,
} Toyama-shi, 930-8555 Japan
There are many kinds of visual illusion, such as Edgar Rubin \& Harrower, Lateral Inhibition, Muller's illusion, Illusion of Depth, Afterimage, Fill up illusion, Perspective illusion, Camouflage illusion, Illusion of Contour, Apparent Motion Perception [7]-[9].

But most of them were discussed qualitatively and therefore their mechanisms have not been elucidated. A new illusion that can be quantitatively measured and quantitatively analyzed is desired. In this paper, we report a new speed illusion that falling speed of descent of snowflakes seen from the partially blinds window looks quite quicker than that of snowflakes seen from the window without a blind. We call it 'Blind Illusion (BI)' that is found by one of authors who looked outside through a window in a snow day. We reappear the nature phenomenon in a computer, shown in Fig. 1, and confirm the new type speed illusion. In this figure, the white spots indicate snowflakes and dark red long rectangles indicate many blind strips.

Furthermore, we simulate various conditions in nature, such as falling speed of snowflakes, falling direction, width and number of blind strips, and so on, to measured illusion amount of BI quantitatively. Before our research in this paper, Miyajima et al. discussed the topic of apparent movement and pursuit eye movement in BI [10], Jinye et al. discussed the effect of luminance difference on BI [11]. We study the number and width of blind strips sequentially. The results can be expected to the elucidation of the mechanism of visual illusions, as well as leading to the understanding of perceptual features and intelligence.

\section{EXPERIMENT I}

\section{A. Introduction to Experiment}

When we observed natural snowfalls, we found a phenom-

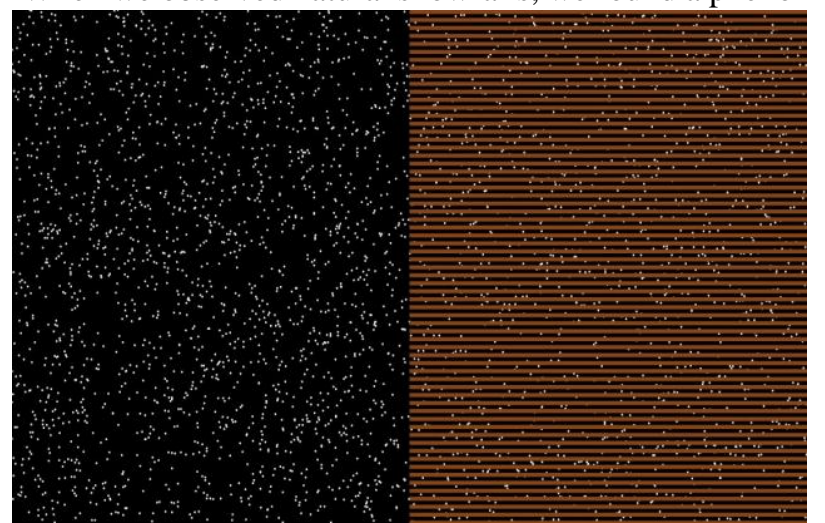

Fig. 1: Blind illusion model. 


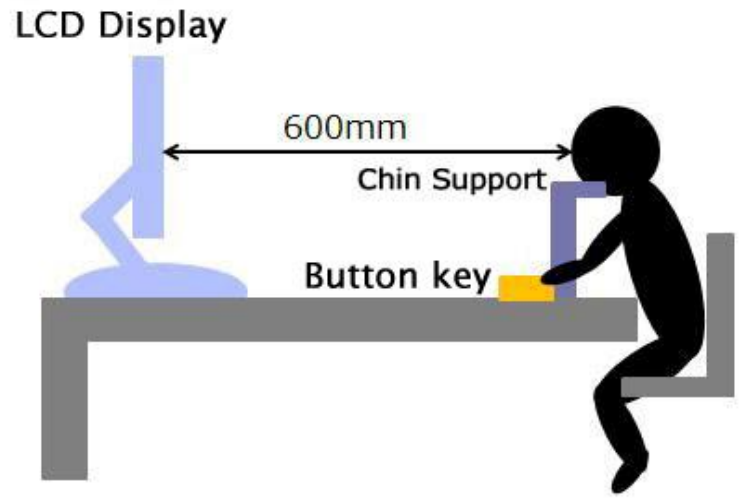

Fig. 2: Experimental equipment.

enon of visual illusion called BI and simulated it in computers. This experiment is based on those previous ones and further simulates all kinds of real situations, then abstracts a model beyond reality in order to test some new research requirements and goals.

In the experiment, we design two windows in which simulating falling of snowflakes to contrast, the left one is called a normal window, and the right is called BI one with some blind strips. We plan to utilize a variety of different data, including the falling speed of snowflakes, the width of blind strips, and the number of blind strips, to test the experimental participants.

We will make quantitative analysis of BI phenomena, detect the key information about the occurrence and change of $\mathrm{BI}$, and speculate the conditions of promoting and inhibiting BI phenomena.

\section{B. Equipment}

The experiment will be carried out in a darkroom which prevents light outside from interfering. The experimental equipment is shown in Fig. 2. A liquid crystal display (LCD), whose parameters are shown in Table 1, is placed on an experimental table, $600 \mathrm{~mm}$ far from a tester sitting on a chair. The head of the tester is fixed with a chin support. The answer device is put near the tester's hand. Ten students aged between 20 and 30 years old of the University of Toyama will take part in this experiment. They are healthy and of good eyesight.

\section{Stimuli}

There are two kinds of stimulus scenes in this experiment. One is standard stimulus. The screen background is black. There are about 2000 white dots with a diameter of $0.1 \mathrm{deg}$ randomly distributed on the picture. These light spots are used to simulate snowflakes with a falling speed of $0.5 \mathrm{deg} / \mathrm{s}$.

The other is comparison stimulus. The screen background is also black and the number, size and location of snowflakes are exactly the same as those in standard stimulus. Some dark red rectangular strips are evenly distributed in the picture to simulate the effect of the blinds, shown in Fig. 3. The dark red

Table 1: Parameters of LCD.

\begin{tabular}{c|c}
\hline Type & LCD-AD172SEB \\
\hline Size & 17 inch \\
\hline Maximum range & $338 \mathrm{~mm} \times 270 \mathrm{~mm}$ \\
\hline Resolution & 1280 pixel $\times 1024$ pixel \\
\hline Maximum refresh frequency & $75 \mathrm{~Hz}$ \\
\hline
\end{tabular}

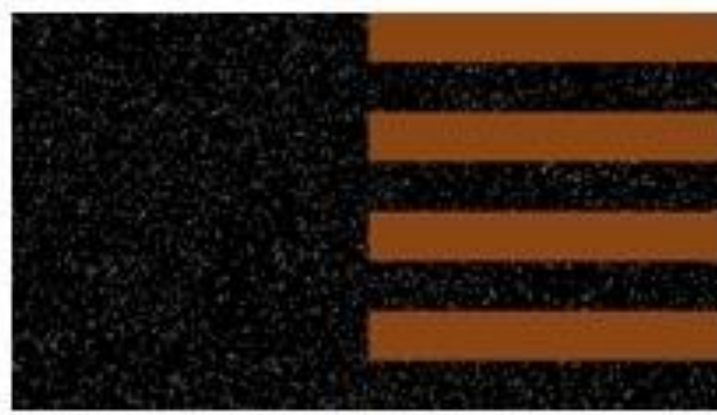

Fig. 3: An example of stimuli.

Table 2: Parameters of Comparison Stimulus.

\begin{tabular}{c|c|c|c|c|c|c}
\hline $\mathrm{n}$ & 1 & 2 & 3 & $\ldots$ & 9 & 10 \\
\hline $\begin{array}{c}\text { Number of } \\
\text { blind strips }\end{array}$ & 1 & 2 & 4 & $\ldots$ & 256 & 512 \\
\hline $\begin{array}{c}\text { Width between } \\
\text { two strips (deg) }\end{array}$ & 128 & 64 & 32 & $\ldots$ & 0.5 & 0.25 \\
\hline
\end{tabular}

area is an area where snowflakes cannot be seen, indicating the part that is covered by blind strips. The black area between two blind strips is the visible part of snowflakes, indicating the gap between the two strips. The width of a blind strip is the same as that of the gap between the two strips. The number of blind strips increases by $2 \mathrm{n}-1$, and the width between two strips decreases consequently. The parameters are listed in Table 2.

\section{D.Methods and procedure}

Each participant in the experiment will be tested independently. The tester sits in the chair and adjusts to darkness, and then begins to observe the display screen and presses the button to answer. The display screen is divided vertically into two blocks, the left is standard stimulus area, and the right is comparison stimulus area. Each participant experiences by the given ten pictures, listed in Fig. 4, in which there are more and more blind strips.

The experimental procedure is described as follows: $\mathrm{S} 1: \mathrm{n}=1$;
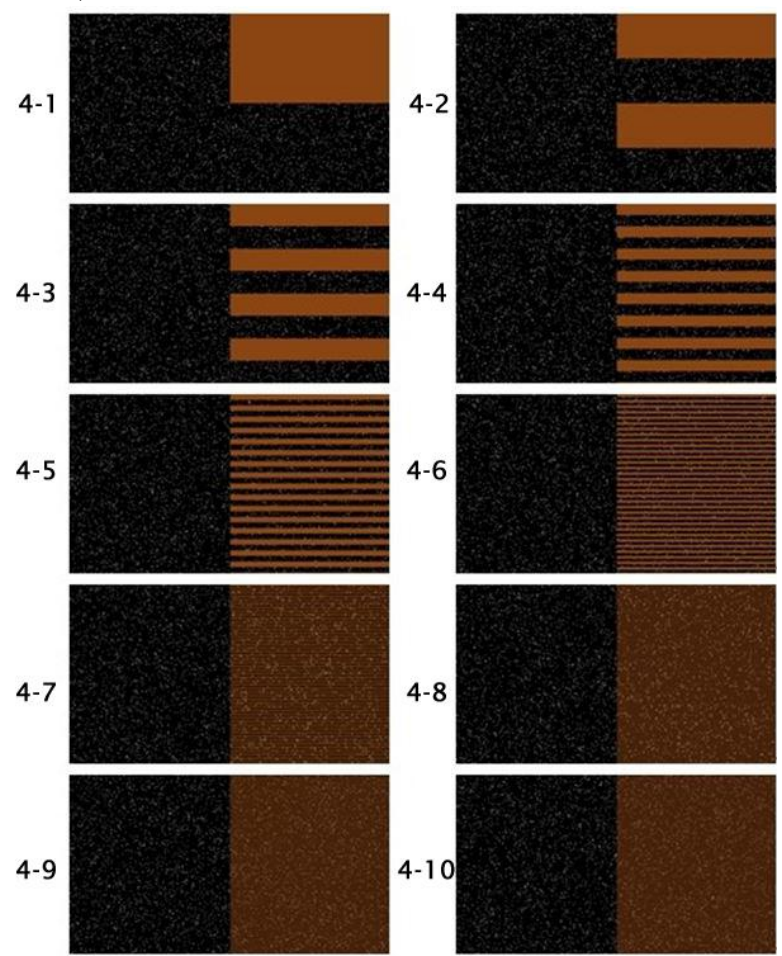

Fig. 4: Stimuli. 
S2: If $\mathrm{n}<11$ then Fig. 4-n appears, else jump to S5;

S3: The tester presses the left button if he feels that the snowflakes in the stimulus area fall faster than the ones in the standard stimulus area;

S4: The test system slows down the falling speed of snowflakes in the comparison stimulus area, then jump to S2; S5: The tester presses the right button if he feels that the snowflakes in the stimulus area fall as fast as the ones in the standard stimulus area;

S6: $n+1=>$ n, jump to $\mathrm{S} 2$;

S7: End.

\section{E. Results and analysis}

We record all the testers' responses and the corresponding system data, then analyze them. Fig. 5 depicts the changes of illusion amount of each tester in different stimuli, in which the horizontal axis indicates the number of blind strips and the vertical axis indicates the illusion amount. In Fig. 5-1, when the number of blind strips is 1 , the first person (P1) judges that the falling speed of snowflakes in comparison stimulus area is the same as that in standard stimulus area. That is a correct judgment, so the illusion amount is 0 at point 1 of horizontal axis and the number of blind strips increases to 2 . When the number increases from 2 to 16 , the illusion amount of P1 remains at 0.1 . Subsequently the errors of P1's judgement become more and more with the number varying from 16 to 128 . And the illusion amount reaches a peak value 1.7

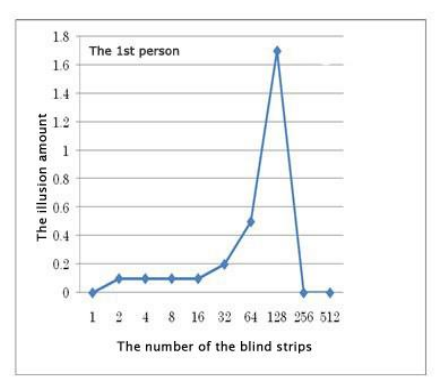

$5-1$

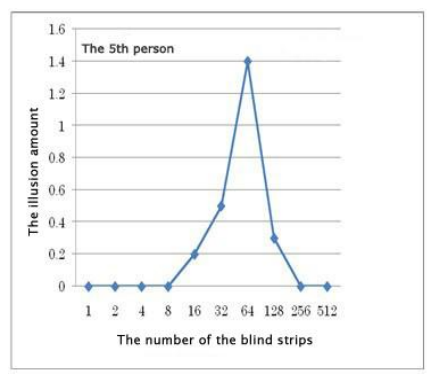

$5-5$

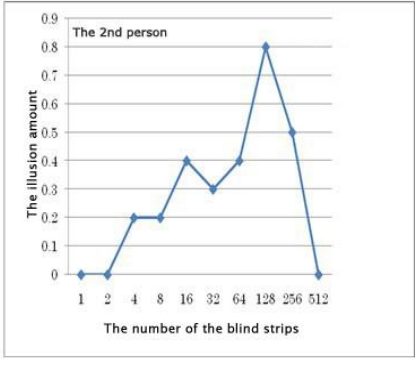

$5-2$

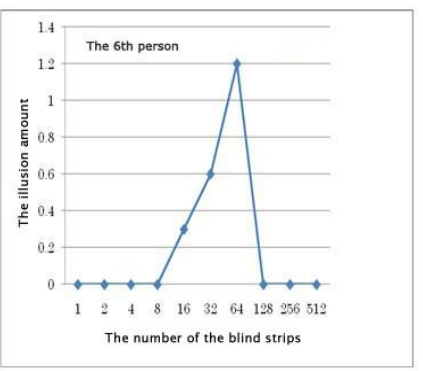

$5-6$

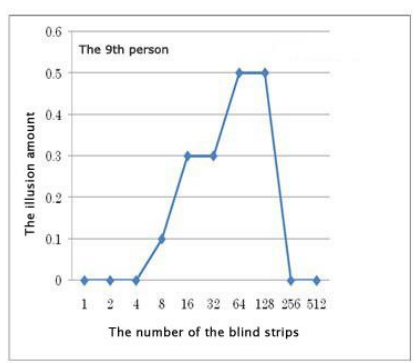

$5-9$

eventually when the number reaches 128 , that is to say, the diameter of a grain of snow is the same as the width of two blind strips. P1's judgements return to be correct when the diameter of a grain of snow is bigger than the width of two blind strips. We can understand the other 9 pictures in the same way.

Considering the ten figures together, we can see that the illusion amount reaches a maximum value generally when the number of blind strip is 64 or 128 . Of the ten participants, six have the maximum illusion amount when the number of blind strips is 64, and two keep the maximum values from 64 to 128 , and the other two reach the peak at 128 .

We synthesize all the data of 10 participants, and then obtain the mean curve, shown in Fig. 6. It can be seen from the figure, all the participants almost have no errors when the value of horizontal axis is 1 ; when the value increases to $2-8$ a few errors appear on these participants and we can infer there do exist visual illusion phenomena; and when the value increases from 8 to 64 the illusion amount rises up sharply, then reaches the highest value at 64 ; while the value continues to increase to 64-512, the illusion amount falls down sharply.

Before this experiment we predicted the changes of illusion amount and the conclusion which is that illusion amount will first increase to a peak value and then fall down with the increasing of the number of blind strips has been verified. Furthermore we get a critical value 64 at which the illusion amount reaches the maximum value.

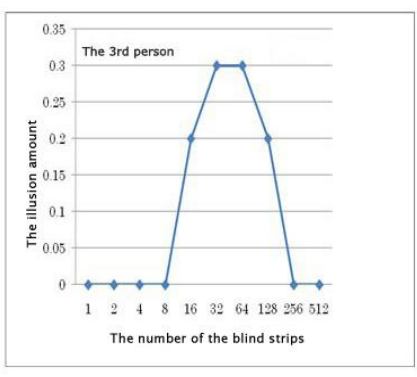

$5-3$

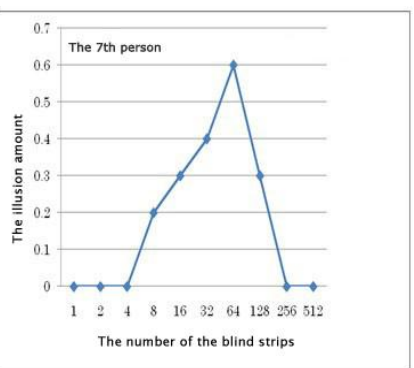

$5-7$

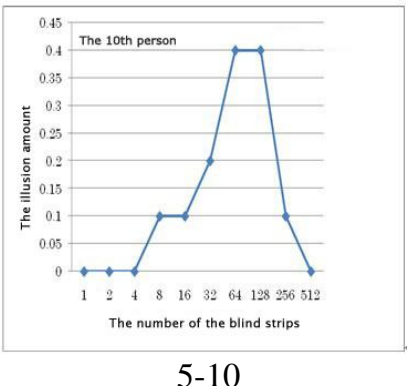

$5-10$

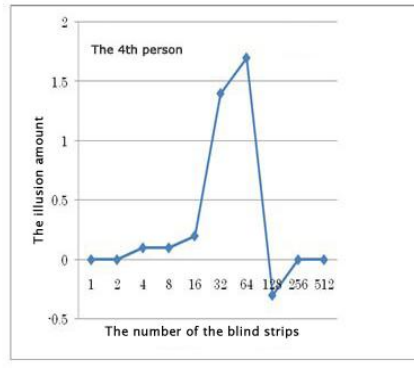

$5-4$

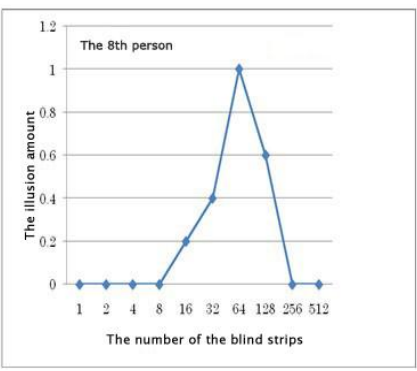

$5-8$

Fig. 5: Personal illusion amount (ten participants). 


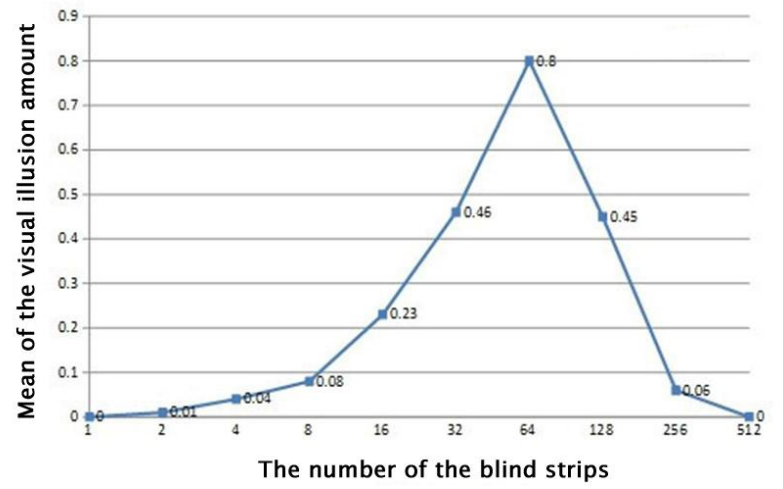

Fig. 6: Mean illusion amount of ten participants.

\section{EXPERIMENT II}

\section{A. Introduction to Experiment}

From experiment I, we can see that almost the illusion all experimenters have the highest illusion amount when the number of blind strips is 64. Therefore, in experiment II, we focus on adjusting the interval width between blind strips while fixing the number of blind strips to be 64 . We observe what changes occur to illusion amount.

\section{B. Equipment}

This experiment will be conducted in a darkroom which is the same as that in Experiment I. And the others are the same as those in the former experiment.

\section{C.Stimuli}

There are also two kinds of stimulus scenes in this experiment. One is standard stimulus. There are about 2000 white dots with a diameter of $0.1 \mathrm{deg}$ randomly distributed on the picture. These light spots are used to simulate snowflakes with a falling speed of $0.5 \mathrm{deg} / \mathrm{s}$.

The other is comparison stimulus. The screen background is also black and the number, size and location of snowflakes are exactly the same as those in standard stimulus. Some dark red rectangular strips are evenly distributed in the picture to simulate the effect of the blinds. It must be pointed out that the number of blinds is fixed to be 64 . However, the interval width between any two blinds are changing from $0.25 \mathrm{deg}$ to $3.75 \mathrm{deg}$.

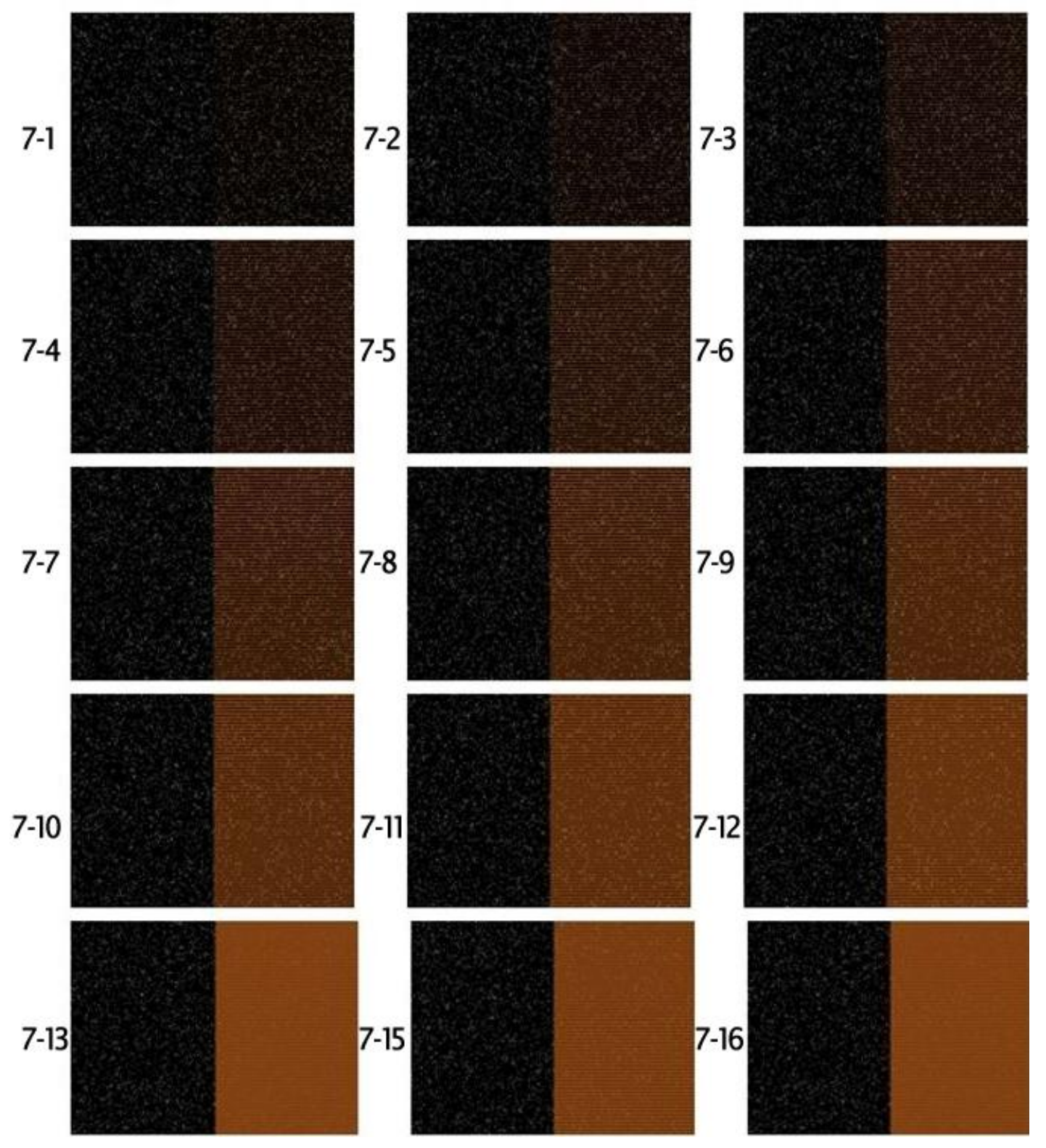

Fig.7: The change of intervals. 


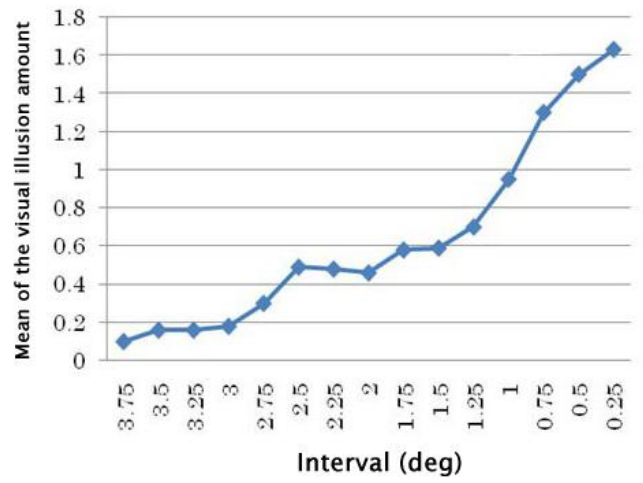

Fig. 8: Mean illusion amount of ten participants of Experiment II.

The participants observe the standard and comparison stimulus area at the same time. The falling speed of snowflakes in both areas keeps the same. We will adjust the interval between blind strips in comparison stimulus until the participants feel the falling speed of snowflake in the standard stimulus is the same as that in the comparison.

\section{D.Methods and procedure}

According to the results of Experiment I, the illusion amount reaches the maximum value when the number of blind strips is 64. Thus in this experiment, we will adjust the interval between blind strips. Due to the limitation of the display screen, the interval will change from 15 pixels to 1 pixel. And then we will examine how the illusion amount changes. The participants are tested separately. The initial intervals are set to 15 pixels and the first person (P1) begin to

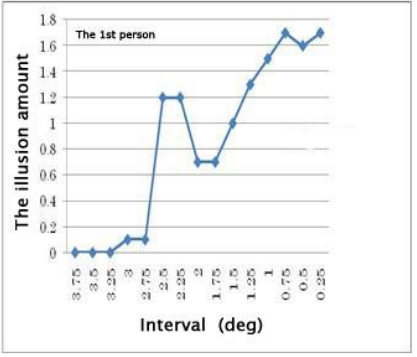

$9-1$

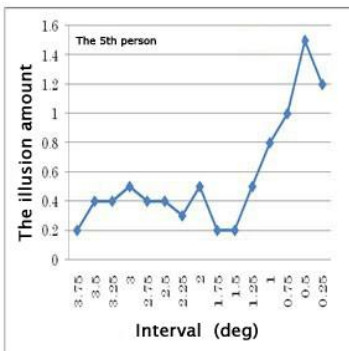

$9-5$

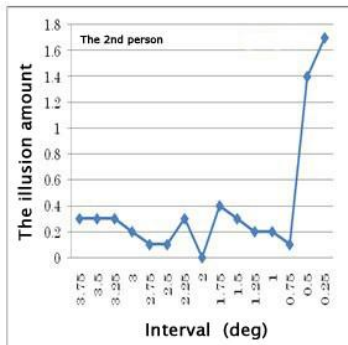

$9-2$

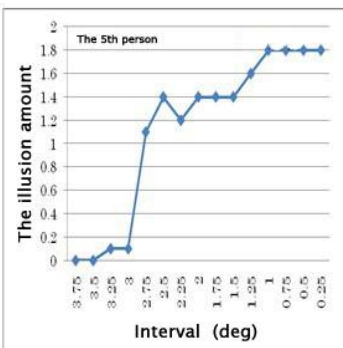

$9-6$

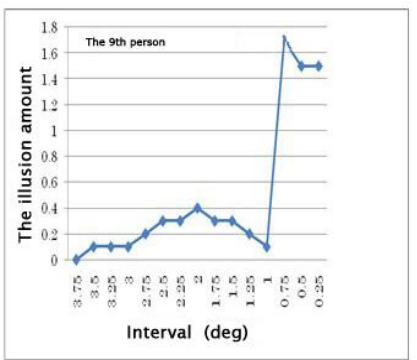

9-9 judge whether the speed in the left is the same as that in the right or not. If the judgement is correct, the intervals will be decrease to 14 pixels and then P1 is asked to judge the two speeds. If the judgement of P1 is wrong, we will adjust the falling speed of snowflakes in the right until P1 feel the two speed are the same. We record the data produced in the experiment. The experiment is carried out in such a way, and the intervals are gradually reduced. When the interval is down to 1 pixel, the experiment ends. The change of intervals is shown in Fig. 7.

\section{E. Results and analysis}

From the mean illusion amount of ten participants in Fig. 8, we can see that there is almost no visual illusion produced when the intervals between blind strips are reduced from $3.75 \mathrm{deg}$ to $3 \mathrm{deg}$, and even if there is, there is almost no change in illusion amount. When the intervals decrease from $3 \mathrm{deg}$ to $2.5 \mathrm{deg}$, the illusion amount increases gradually. However, the illusion amount decreases when the intervals are reduced from $2.5 \mathrm{deg}$ to $2 \mathrm{deg}$. And then it gradually decreases with the decreasing of the intervals.

\section{CONCLUSION}

The rapid development of computer science and technology provides a new technology for the study of visual illusion. Specially, it can simulate natural phenomena and even some scenes that does not exist in nature. The study of visual illusion gradually shifts from theoretical mechanism to social life, such as the applications in road sign, interior dec-

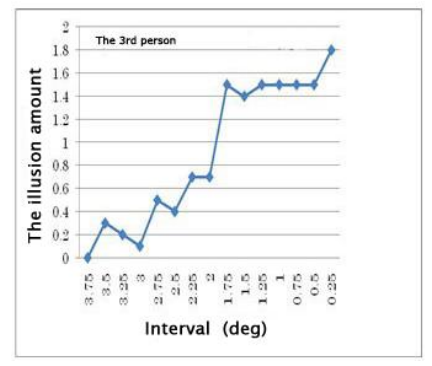

$9-3$

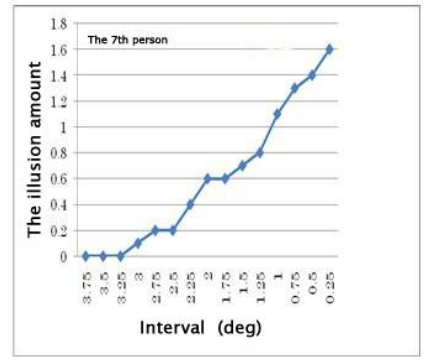

$9-7$

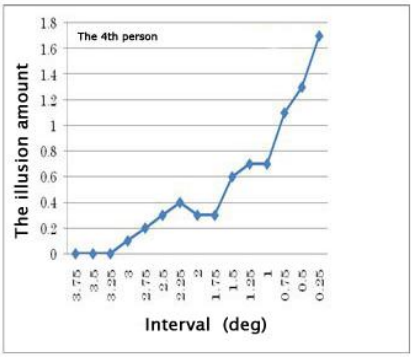

9-4

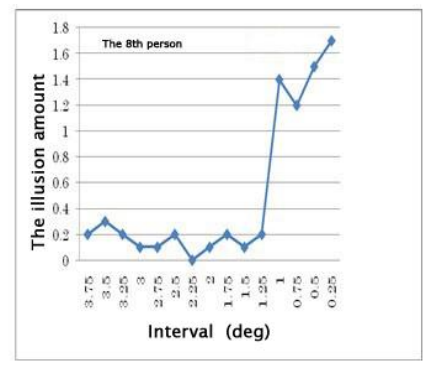

9-8

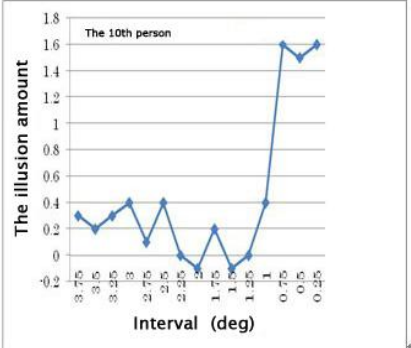

9-10

Fig. 9 Personal illusion amount (ten participants) of Experiment II. 
oration, plastic art, film stunt and so on which can introduce people to make full of visual illusion to improve their life.

The study in this paper is based on a newly discovered speed illusion which is called BI. We reappear the realistic and unrealistic scenes in a computer. We adjust the simulated scenes, and record the answers of the experimental participants about whether the falling speed of snowflakes in the standard stimulus is the same as that in the comparison stimulus or not, and then calculate the illusion amounts. Through this study, we can conclude that how the number of blind strips, the interval of blind strips, and the falling speed of snowflakes affect the illusion amount, and furthermore analyze the specific critical values at which the BI happens and the illusion amount reaches the peak, respectively. We also can construct mathematical models, utilize computers to simulate all kinds of scenes, understand the theories of visual illusion better, and master some methods of analyzing BI.

\section{REFERENCES}

[1] W.A. Simpson, U. Shahani, and V. Manahilov, "Illusory percepts of moving patterns due to discrete temporal sampling," Neuroscience letters, vol.375, no.1, pp.23-27, 2005.

[2] S.J. Anderson and R.F. Hess, "Post-receptoral undersampling in normal human peripheral vision," Vision Research, vol.30, no.10, pp.1507-1515, 1990.

[3] T.J. Andrews and A.N. McCoy, "Can illusory motion disrupt tracking real motion?," Perception, vol.26, no.3, pp.269-275, 1997.

[4] D. Finlay, P. Dodwell, and T. Caelli, "The waggon-wheel effect," Perception, vol.13, no.3, pp.237-237, 1984.

[5] A. Fraser and K.J. Wilcox, "Perception of illusory movement," Nature, vol.281, no.5732, p.565, 1979 .

[6] J. Faubert and A.M. Herbert, "The peripheral drift illusion: A motion illusion in the visual periphery," Perception, vol.28, no.5, pp.617-621, 1999.

[7] D.M. Eagleman, "Visual illusions and neurobiology," Nature Reviews Neuroscience, vol.2, no.12, p.920, 2001.

[8] B.C. Kong and A. Professor, "Comparison between human vision and computer vision," Nature Magazine, 2002.

[9] A. Nieder, "Seeing more than meets the eye: processing of illusory contours in animals," Journal of Comparative Physiology A, vol.188, no.4, pp.249-260, 2002.

[10] M. Ryuushou, Z. Tang, and Y. Kazuya, "The influence of the pseudo-motion and the par-chute eye movement in blind illusion," Toyama, Toyama University, 2014. Thesis. [Published in Japanese].

[11] T. Junichi, Z. Tang, and Y. Kazuya, "Basic research of a speed optical illusion by a blind (the influence luminance difference exerts)," Toyama, Toyama University, 2015. Thesis. [Published in Japanese].
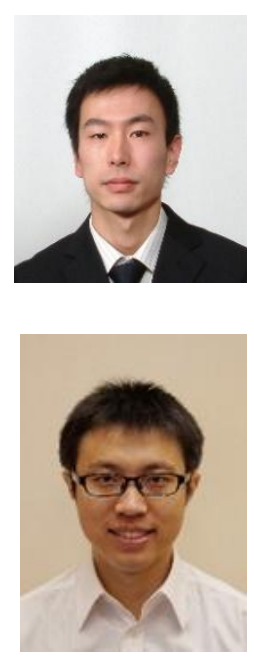

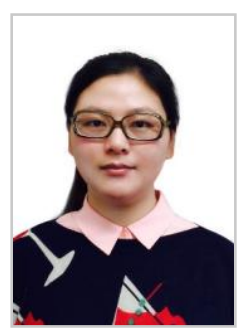

Jian Sun received the B.S. degree from Nanjing Normal University, Nanjing, China in 2000 and an M.S. degree from Yangzhou University, Yangzhou, China, in 2010. Now she is working toward the D.E. degree at University of Toyama, Toyama, Japan Her main research interests are computational intelligence, multiple-valued logic, artificial neural networks and optical information processing.

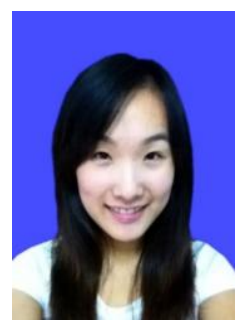

Xiaoxiao Qian received the B.S. degree from Dalian Neusoft University of Information, Liaoning, China in 2013, and the M.S. degree from University of Toyama, Toyama, Japan in 2017. Now, she is currently working toward the Ph.D. degree at University of Toyama, Toyama, Japan. Her main research interests include data mining, computational intelligence, swarm intelligence algorithms, artificial neural networks and optical information processing.

Yining Tong received the B.S. degree and the M.S. degree from the University of Toyama, Toyama, Japan, in 2013 and 2015, respectively. Now he is working toward the D.E. degree at University of Toyama, Toyama, Japan. His main research interests are optical information processing, computational intelligence and artificial neural networks.

Hiroyoshi Todo received the B.S. degree from the University of Toyama, Toyama, Japan, in 2016. Now he is working in Wicresoft Japan Inc., Tokyo, Japan. His main research interests are perception information processing, computational intelligence and artificial neural networks. 